\title{
A Novel Multistage Equalization Algorithm
}

\author{
Pei Xiao, Rolando Carrasco \\ School of Electrical, Electronic and Computer Engineering \\ University of Newcastle Upon Tyne NE1 7RU, United Kingdom \\ E-mail: pei.xiao, r.carrasco@ncl.ac.uk \\ Ian Wassell \\ Computer Laboratory, University of Cambridge \\ 15 JJ Thomson Avenue, CB3 OFD, United Kingdom \\ E-mail: i jw24@eng.cam.ac.uk
}

\begin{abstract}
A novel equalization algorithm utilizing improper nature of the intersymbol interference (ISI) is introduced in this paper. We show that full exploitation of the available information on the second-order statistics of the observed signal entails widely linear processing and that previously known linear minimum mean square error (MMSE) equalizers represent sub-optimum solutions. The proposed scheme is generally applicable for both real and complex signal constellations. The results show that accounting for the improper nature of the ISI leads to significant performance gain compared to conventional equalization schemes.
\end{abstract}

\section{INTRODUCTION}

For a complex random vector $\mathbf{r}$, its second-order properties are completely characterized by its autocorrelation matrix $\mathbf{C}=$ $\mathrm{E}\left[\mathbf{r} \mathbf{r}^{H}\right]^{\dagger}$ as well as the pseudo-autocorrelation matrix $\tilde{\mathbf{C}}=$ $\mathrm{E}\left[\mathbf{r r}^{T}\right][1]$. Most existing studies on receiver algorithms only exploit the information contained in the autocorrelation function of the observed signal. The pseudo-autocorrelation matrix $\tilde{\mathbf{C}}$ is usually not considered and is implicitly assumed to be zero. While this is the optimum strategy when dealing with proper complex random processes (i.e., when pseudo-autocorrelation $\tilde{\mathbf{C}}$ is vanishing) [2], it turns out to be sub-optimum in situations where the transmitted signals and/or interference are improper complex random processes (i.e., $\tilde{\mathbf{C}}$ is non-vanishing), for which the performance of a linear receiver can be improved by the use of widely linear processing (WLP) [3]. It was shown in [1] that the performance gain of WLP compared to conventional processing in terms of mean square error can be as large as a factor of 2 .

The concept of WLP has been used in several papers for enhancing the performance of equalizers which are used to combat the intersymbol interference (ISI) induced by frequencyselective multipath channels. For example, it was applied to the equalization of real-valued data transmitted over ISI channels having complex-valued channel coefficients [4]; and applied to the equalization of space-time block encoded transmissions over multiple-input, multiple-output (MIMO) channels [5]. It has been shown that the performance of a linear minimum mean square error (MMSE) equalizer may be improved significantly, if not only the original but also the complex conjugated version of the received signal are jointly processed and linearly combined. A MMSE equalizer and a decisionfeedback equalizer (DFE) employing WLP and implemented via FIR filters for a MIMO frequency selective channel were

\footnotetext{
${ }^{\dagger}$ The superscript operators ()$^{H},()^{*},()^{T}$ denote the conjugate transpose, conjugate, and transpose operations, respectively.
}

proposed in [6], [7]. It was concluded that the use of WLP yields considerable performance improvements at the cost of only a limited increase in complexity compared to conventional linear processing. In order to achieve a gain compared to the conventional approach, either the pseudo-autocorrelation of the observation or the pseudo-crosscorrelation between observation and desired variable must be nonzero. An important example for such a scenario is the transmission of real-valued data over a complex-valued channel [4].

Linear MMSE filter based turbo equalization which combines equalization and decoding in an iterative fashion has previously been studied, e.g., in [8]-[10] where only the information contained in the autocorrelation function of the observations was employed. In this paper, we further develop the algorithm presented in [8]-[10] by utilizing the improperness of the ISI and exploiting the information contained in the pseudoautocorrelation function of the observations. In contrast to most previous studies concerning widely linear (WL) equalization which have been mainly confined to real-valued signals (one exception is the work presented in [5] where equalizers with WLP were designed for complex signals which become improper by space-time block coding), the proposed equalization algorithm can be applied to systems with real or complex modulation schemes without space-time coding. Section II describes how improper ISI is utilized in the design of equalization schemes; while in Section III, the proposed scheme is evaluated over some static and time-varing ISI channels. The results show that accounting for the improper nature of the ISI leads to better performance than conventional linear MMSE equalization schemes.

\section{EQUALIZATION USING IMPROPER ISI}

The transmission system under study will now be briefly described. For a system without channel coding, the information sequence $\left\{b_{n}\right\}$ is directly mapped into PSK/QAM symbols $\left\{s_{n}\right\}$, which are transmitted over a multipath frequency selective fading channel with $L$ resolvable paths, having complex channel gains $h_{0}, h_{1}, \ldots, h_{L-1}$. The received signal can be expressed as

$$
r_{n}=\sum_{l=0}^{L-1} s_{n-l} h_{l}+v_{n},
$$

where $h_{l}$ is the complex channel coefficient and is assumed to remain constant during the transmission of one block of data. The complex additive white Gaussian noise (AWGN) $v_{n}$ 
with zero mean and variance $N_{0}$ is assumed to be proper. The proposed equalization algorithm works for both real and complex signals. For simplicity, we employ BPSK/QPSK as real/complex-valued modulation scheme in this work. However, its extension to higher level ASK (amplitude-shift keying) and PSK/QAM schemes is straightforward. The transmitted symbol at time instant $n$ is denoted as $s_{n}$. For BPSK modulation, $s_{n}$ is real-valued; for QPSK modulation, we denote $s_{n}=x_{n}+j y_{n}$, where $x_{n}, y_{n}=\frac{ \pm 1}{\sqrt{2}}$.

The task of the receiver is to detect the transmitted information bits $\left\{b_{n}\right\}$ given the received observation $\left\{r_{n}\right\}$. To this end, we need first to detect the transmitted symbols $\left\{s_{n}\right\}$ which are corrupted with ISI and AWGN. An equalizer is required to reduce the detrimental effect of ISI. Here, we consider the equalization algorithm presented in [8]-[10] and develop an enhanced scheme by modifying the MMSE filter design criterion and applying WLP. According to (1), the interference canceled version of the received vector is given as [8], [9]

$$
\mathbf{r}_{n}^{\prime}=\mathbf{r}_{n}-\mathbf{H} \overline{\mathbf{s}}_{n}=\mathbf{H}\left[\mathbf{s}_{n}-\overline{\mathbf{s}}_{n}\right]+\mathbf{v}_{n}
$$

where $\quad \mathbf{r}_{n}=\left[\begin{array}{llll}r_{n} & r_{n+1} & \ldots & r_{n+L-1}\end{array}\right]^{T}, \quad \mathbf{v}_{n}=$ $\left[\begin{array}{llll}v_{n} & v_{n+1} & \ldots & v_{n+L-1}\end{array}\right]^{T}$ denote the received vector and the noise vector, respectively; and $\mathbf{r}_{n}^{\prime}$ is the ISI canceled version of $\mathbf{r}_{n}$. The vectors $\mathbf{s}_{n}$ and $\overline{\mathbf{s}}_{n}$ are defined respectively as $\mathbf{s}_{n}=\left[\begin{array}{lllllll}s_{n-L+1} & \ldots & s_{n-1} & s_{n} & s_{n+1} & \ldots & s_{n+L-1}\end{array}\right]^{T}$, $\overline{\mathbf{s}}_{n}=\left[\begin{array}{lllllll}\bar{s}_{n-L+1} & \ldots & \bar{s}_{n-1} & 0 & \bar{s}_{n+1} & \ldots & \bar{s}_{n+L-1}\end{array}\right]^{T}$, where the latter contains the estimate of the interference symbols from the previous iteration. The derivation of $\overline{\mathbf{s}}_{n}$ will be given later on. The channel matrix is defined as

$$
\mathbf{H}=\left[\begin{array}{cccccccc}
h_{L-1} & h_{L-2} & \ldots & h_{0} & 0 & 0 & \ldots & 0 \\
0 & h_{L-1} & \ldots & h_{1} & h_{0} & 0 & \ldots & 0 \\
\vdots & \vdots & \vdots & & & & & \\
0 & 0 & 0 & \ldots & h_{L-1} & h_{L-2} & \ldots & h_{0}
\end{array}\right]
$$

Note that (2) represents a decision-directed iterative scheme, where the detection procedure at the the $n^{t h}$ stage uses the symbol estimates from the $(n-1)^{t h}$ stage. The performance is improved in an iterative manner owing to the fact that the symbols are more accurately estimated (meaning better interference cancellation), as the iteration procedure goes on. For simplicity, the iteration index is omitted, whenever no ambiguity arises.

In order to further suppress the residual interference in $\mathbf{r}_{n}^{\prime}$, an instantaneous linear MMSE filter is applied to $\mathbf{r}_{n}^{\prime}$, to obtain [8], [9] $z_{n}=\mathbf{w}_{n}^{H} \mathbf{r}_{n}^{\prime}$, where the filter coefficient vector $\mathbf{w}_{n}$ is chosen to minimize $e_{n}^{\mathrm{L}}=\mathrm{E}\left\{\left[\mathbf{w}_{n}^{H} \mathbf{r}_{n}^{\prime}-s_{n}\right]^{2}\right\}$. Refer to [8]-[10] for a detailed description of the conventional MMSE algorithm. Next, we shall discuss how the performance can be improved by applying WLP, the principle of which is not only to process $\mathbf{r}_{n}^{\prime}$, but also its conjugated version $\mathbf{r}_{n}^{* *}$ in order to derive the filter output, i.e., $z_{n}=\boldsymbol{\omega}_{n}^{1} \mathbf{r}_{n}^{\prime}+\boldsymbol{\omega}_{n}^{2} \mathbf{r}_{n}^{\prime *}=\boldsymbol{\omega}_{n}^{H} \mathbf{y}_{n}$, where $\boldsymbol{\omega}_{n}=$ $\left[\begin{array}{ll}\boldsymbol{\omega}_{n}^{1} & \boldsymbol{\omega}_{n}^{2}\end{array}\right]^{H}$ and $\mathbf{y}_{n}=\left[\begin{array}{ll}\mathbf{r}_{n}^{\prime} & \mathbf{r}_{n}^{\prime *}\end{array}\right]^{T}$. Substituting (2) into the above equation yields

$$
\begin{aligned}
z_{n} & =\boldsymbol{\omega}_{n}^{1} \mathbf{H}\left[\mathbf{s}_{n}-\overline{\mathbf{s}}_{n}\right]+\boldsymbol{\omega}_{n}^{1} \mathbf{v}_{n}+\boldsymbol{\omega}_{n}^{2} \mathbf{H}^{*}\left[\mathbf{s}_{n}^{*}-\overline{\mathbf{s}}_{n}^{*}\right]+\boldsymbol{\omega}_{n}^{2} \mathbf{v}_{n}^{*} \\
& =\boldsymbol{\omega}_{n}^{1} \mathbf{h} s_{n}+\boldsymbol{\omega}_{n}^{2} \mathbf{h}^{*} s_{n}^{*}+\mathbf{w}_{n},
\end{aligned}
$$

where $\mathbf{h}=\left[\begin{array}{llll}h_{0} & h_{1} & \ldots & h_{L-1}\end{array}\right]^{T}$. The decision statistic $z_{n}$ in (3) contains the scaled version of the symbol $s_{n}$ and its conjugate $s_{n}^{*}$ as well as the combined interference cancellation residual and noise denoted as $\mathbf{w}_{n}$. Accounting for this changes at the filter output $z_{n}$, the cost function needs to be revised accordingly as

$$
e_{n}^{\mathrm{WL}}=\mathrm{E}\left[\left|z_{n}-s_{n}-\gamma s_{n}^{*}\right|^{2}\right]=\mathrm{E}\left[\left|\boldsymbol{\omega}_{n}^{H} \mathbf{y}-s_{n}-\gamma s_{n}^{*}\right|^{2}\right] .
$$

For real-valued constellations (the symbol $s_{n}$ is real-valued), $\gamma=1$ is appropriate so that $e_{n}^{\mathrm{WL}}=\mathrm{E}\left[\left|\boldsymbol{\omega}_{n}^{H} \mathbf{y}-s_{n}-s_{n}^{*}\right|^{2}\right]=$ $\mathrm{E}\left[\left|\boldsymbol{\omega}_{n}^{H} \mathbf{y}-2 s_{n}\right|^{2}\right]$. In this case, $\boldsymbol{\omega}_{n}^{2}=\boldsymbol{\omega}_{n}^{1 *}$ [3], [4], and therefore, $e_{n}^{\mathrm{WL}}=\mathrm{E}\left[\operatorname{Re}\left\{\boldsymbol{\omega}_{n}^{1} \mathbf{r}_{n}^{\prime}\right\}-s_{n}\right](\operatorname{Re}\{\cdot\}$ and $\operatorname{Im}\{\cdot\}$ denote the real and imaginary part of a complex variable, respectively), which is a better cost function than $e_{n}^{\mathrm{L}}=\mathrm{E}\left\{\left[\mathbf{w}_{n}^{H} \mathbf{r}_{n}^{\prime}-s_{n}\right]^{2}\right\}$, since a conventional MMSE filter yields a complex-valued filter output; however, only the real part of this output is relevant for the decision for systems with a real-valued constellation. It was shown in [4] that $e_{n}^{\mathrm{WL}}<e_{n}^{\mathrm{L}}$, leading to an equalizer with enhanced performance. For complex-valued constellations, the role of the parameter $\gamma$ (the value of which should not be 1) will become apparent in Section III. It is worth noticing that the conventional linear MMSE equalizer is a special case of the WL equalizer, when $\boldsymbol{\omega}_{n}^{1}=\mathbf{w}_{n}^{H}$ and $\boldsymbol{\omega}_{n}^{2}=\mathbf{0}$. The WL equalizers are expected to exhibit better performance than their linear counterparts. In particular, the conditions under which WL estimator can yield significant improvements over linear ones are detailed in [3]. By expanding (4), we obtain

$$
\begin{aligned}
e_{n}^{\mathrm{WL}}= & \mathrm{E}\left[\left|\boldsymbol{\omega}_{n}^{H} \mathbf{y}-s_{n}-\gamma s_{n}^{*}\right|^{2}\right]=\boldsymbol{\omega}_{n}^{H} \mathbf{C}_{\mathbf{y y}} \boldsymbol{\omega}_{n}-\gamma \boldsymbol{\omega}_{n}^{H} \tilde{\mathbf{C}}_{\mathbf{y} s} \\
& -\boldsymbol{\omega}_{n}^{H} \mathbf{C}_{\mathbf{y} s}-\mathbf{C}_{s \mathbf{y}} \boldsymbol{\omega}_{n}-\gamma \tilde{\mathbf{C}}_{s \mathbf{y}}^{*} \boldsymbol{\omega}_{n}+1+\gamma^{2},
\end{aligned}
$$

where

$$
\begin{aligned}
& \mathbf{C}_{\mathbf{y y}}=\mathrm{E}\left\{\mathbf{y}_{n} \mathbf{y}_{n}^{H}\right\}=\mathrm{E}\left\{\left[\begin{array}{c}
\mathbf{r}_{n}^{\prime} \\
\mathbf{r}_{n}^{\prime *}
\end{array}\right]\left[\begin{array}{ll}
\mathbf{r}_{n}^{\prime H} & \mathbf{r}_{n}^{\prime T}
\end{array}\right]\right\}=\left[\begin{array}{ll}
\mathbf{C}_{\mathbf{r}^{\prime} \mathbf{r}^{\prime}} & \tilde{\mathbf{C}}_{\mathbf{r}^{\prime} \mathbf{r}^{\prime}} \\
\tilde{\mathbf{C}}_{\mathbf{r}^{\prime} \mathbf{r}^{\prime}}^{*} & \mathbf{C}_{\mathbf{r}^{\prime} \mathbf{r}^{\prime}}^{*}
\end{array}\right] \\
& =\left[\begin{array}{cc}
\mathbf{H} \mathbf{V}_{n} \mathbf{H}^{H}+N_{0} \mathbf{I} & \mathbf{H} \tilde{\mathbf{V}}_{n} \mathbf{H}^{T} \\
\mathbf{H}^{*} \tilde{\mathbf{V}}_{n}^{*} \mathbf{H}^{H} & \mathbf{H}^{*} \mathbf{V}_{n} \mathbf{H}^{T}+N_{0} \mathbf{I}
\end{array}\right] ; \\
& \mathbf{V}_{n}=\mathrm{E}\left\{\left[\mathbf{s}_{n}-\overline{\mathbf{s}}_{n}\right]\left[\mathbf{s}_{n}-\overline{\mathbf{s}}_{n}\right]^{H}\right\}
\end{aligned}
$$

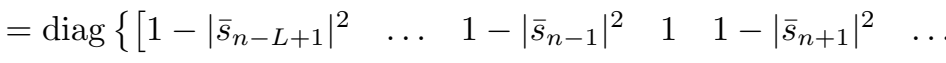

$$
\begin{aligned}
& \mathbf{C}_{\mathbf{y} s}=\mathbf{C}_{s \mathbf{y}}^{H}=\mathrm{E}\left\{\mathbf{y} s^{*}\right\}=\mathrm{E}\left\{\left[\begin{array}{c}
\mathbf{r}^{\prime} \\
\mathbf{r}^{*}
\end{array}\right] s_{n}^{*}\right\}=\left[\begin{array}{c}
\mathbf{C}_{\mathbf{r}^{\prime} s} \\
\tilde{\mathbf{C}}_{\mathbf{r}^{\prime} s}^{*}
\end{array}\right]=\left[\begin{array}{l}
\mathbf{h} \\
\mathbf{0}
\end{array}\right] ; \\
& \mathbf{C}_{s \mathbf{y}}=\mathrm{E}\left[s_{n} \mathbf{y}^{H}\right]=\mathrm{E}\left\{s_{n}\left[\begin{array}{ll}
\mathbf{r}^{\prime H} & \mathbf{r}^{\prime T}
\end{array}\right]\right\}=\left[\begin{array}{llllll}
h_{0}^{*} & h_{1}^{*} & h_{2}^{*} & 0 & 0 & 0
\end{array}\right] ; \\
& \tilde{\mathbf{C}}_{s \mathbf{y}}=\mathrm{E}\left[s_{n} \mathbf{y}^{T}\right]=\mathrm{E}\left\{s_{n}\left[\begin{array}{ll}
\mathbf{r}^{\prime T} & \mathbf{r}^{\prime H}
\end{array}\right]\right\}=\left[\begin{array}{ll}
\mathbf{0} & \mathbf{h}^{H}
\end{array}\right] ; \\
& \tilde{\mathbf{C}}_{\mathbf{y} s}=\tilde{\mathbf{C}}_{s \mathbf{y}}^{T}=\mathrm{E}\left[\mathbf{y} s_{n}\right]=\mathrm{E}\left\{\left[\begin{array}{c}
\mathbf{r}^{\prime} \\
\mathbf{r}^{\prime *}
\end{array}\right] s_{n}\right\}=\left[\begin{array}{c}
\mathbf{0} \\
\mathbf{h}^{*}
\end{array}\right] \text {. }
\end{aligned}
$$

For real-valued constellation, $\tilde{\mathbf{V}}_{n}=\mathrm{E}\left\{\left[\mathbf{s}_{n}-\overline{\mathbf{s}}_{n}\right]\left[\mathbf{s}_{n}-\overline{\mathbf{s}}_{n}\right]^{T}\right\}=$ $\mathbf{V}_{n}$; for complex-valued constellation,

$$
\begin{aligned}
& \tilde{\mathbf{V}}_{n}=\operatorname{diag}\left\{\left[\operatorname{Im}^{2}\left\{\bar{s}_{n-L+1}\right\}-\operatorname{Re}^{2}\left\{\bar{s}_{n-L+1}\right\} \quad \ldots\right.\right. \\
& \operatorname{Im}^{2}\left\{\bar{s}_{n-1}\right\}-\operatorname{Re}^{2}\left\{\bar{s}_{n-1}\right\} \quad 0 \quad \operatorname{Im}^{2}\left\{\bar{s}_{n+1}\right\}-\operatorname{Re}^{2}\left\{\bar{s}_{n+1}\right\} \\
& \left.\left.\ldots \operatorname{Im}^{2}\left\{\bar{s}_{n+L-1}\right\}-\operatorname{Re}^{2}\left\{\bar{s}_{n+L-1}\right\}\right]\right\} .
\end{aligned}
$$

Equation (7) is obtained based on the fact that the real and imaginary parts of $\bar{s}_{n}$ correspond to two independent bits, and are thus uncorrelated, i.e., $\operatorname{E}\left[\operatorname{Re}\left\{s_{n}\right\} \operatorname{Im}\left\{s_{n}\right\}\right]=$ $\operatorname{Re}\left\{\bar{s}_{n}\right\} \operatorname{Im}\left\{\bar{s}_{n}\right\}$. Therefore, for each element of $\tilde{\mathbf{V}}_{n}$

$$
\begin{aligned}
\mathrm{E} & {\left[\left(s_{n}-\bar{s}_{n}\right)^{2}\right]=\mathrm{E}\left\{\left[\left(\operatorname{Re}\left\{s_{n}\right\}+j \operatorname{Im}\left\{s_{n}\right\}\right)-\left(\operatorname{Re}\left\{\bar{s}_{n}\right\}+j \operatorname{Im}\left\{\bar{s}_{n}\right\}\right)\right]^{2}\right\} } \\
= & \mathrm{E}\left[\left(\operatorname{Re}\left\{s_{n}\right\}+j \operatorname{Im}\left\{s_{n}\right\}\right)^{2}-2\left(\operatorname{Re}\left\{\bar{s}_{n}\right\}+j \operatorname{Im}\left\{\bar{s}_{n}\right\}\right)^{2}\right. \\
& \left.+\left(\operatorname{Re}\left\{\bar{s}_{n}\right\}+j \operatorname{Im}\left\{\bar{s}_{n}\right\}\right)^{2}\right] \\
= & \operatorname{Im}^{2}\left\{\bar{s}_{n}\right\}-\operatorname{Re}^{2}\left\{\bar{s}_{n}\right\}+2 j\left[\operatorname{E}\left(\operatorname{Re}\left\{s_{n}\right\} \operatorname{Im}\left\{s_{n}\right\}\right)-\operatorname{Re}\left\{\bar{s}_{n}\right\} \operatorname{Im}\left\{\bar{s}_{n}\right\}\right] \\
= & \operatorname{Im}^{2}\left\{\bar{s}_{n}\right\}-\operatorname{Re}^{2}\left\{\bar{s}_{n}\right\} .
\end{aligned}
$$


Denoting the log-likelihood ratio (LLR) value of $s_{n}$ as $\lambda\left(s_{n}\right)=\lambda\left(x_{n}\right)+j \lambda\left(y_{n}\right)$ for QPSK signals, the soft estimate of $s_{n}$ is computed according to its LLR value $\lambda\left(s_{n}\right)$ as

$\bar{s}_{n}= \begin{cases}\tanh \left(\lambda\left(s_{n}\right) / 2\right) & \text { for BPSK } \\ \tanh \left[\lambda\left(x_{n}\right) / 2\right] / \sqrt{2}+j \tanh \left[\lambda\left(y_{n}\right) / 2\right] / \sqrt{2} & \text { for QPSK }\end{cases}$

At the initial stage, no priori information about $s_{n}$ is available, its LLR value is thus assumed to be zero. Therefore $\bar{s}_{n}=$ 0 , and consequently, $\tilde{\mathbf{V}}_{n}=\mathbf{0}$ and the pseudo-autocorrelation matrix is vanishing $\left(\tilde{\mathbf{C}}_{\mathbf{r}^{\prime} \mathbf{r}^{\prime}}=\mathrm{E}\left[\mathbf{r}_{n}^{\prime} \mathbf{r}_{n}^{\prime T}\right]=\mathbf{H} \tilde{\mathbf{V}}_{n} \mathbf{H}^{T}=\mathbf{0}\right)$ for QPSK signals. Note that the ISI is always improper for BPSK modulated systems since $\tilde{\mathbf{V}}_{n}=\mathbf{V}_{n}$, and $\tilde{\mathbf{C}}_{\mathbf{r}^{\prime} \mathbf{r}^{\prime}}=\mathbf{H V}_{n} \mathbf{H}^{T} \neq$ $\mathbf{0}$ holds also at the initial stage. At the subsequent stages, the ISI terms become improper for both real and complexvalued constellations since $\tilde{\mathbf{C}}_{\mathbf{r}^{\prime} \mathbf{r}^{\prime}}$ is non-vanishing due to a non-vanishing matrix $\tilde{\mathbf{V}}_{n}$. Utilizing the improperness of the ISI signals will consequently lead to a better performance.

Differentiating $\epsilon_{n}^{\mathrm{WL}}$ in (5) with respect to $\boldsymbol{\omega}_{n}$ results in $\frac{\partial e}{\partial \boldsymbol{\omega}_{n}}=\left(\mathbf{C}_{\mathbf{y y}} \boldsymbol{\omega}_{n}\right)^{*}-\mathbf{C}_{s \mathbf{y}}^{T}-\gamma \tilde{\mathbf{C}}_{s \mathbf{y}}^{H}$, which is set to zero to yield the optimum vector of $\boldsymbol{\omega}_{n}$

$\boldsymbol{\omega}_{n}=\mathbf{C}_{\mathbf{y y}}^{-1}\left(\mathbf{C}_{s \mathbf{y}}^{H}+\gamma \tilde{\mathbf{C}}_{s \mathbf{y}}^{T}\right)=\mathbf{C}_{\mathbf{y y}}^{-1}\left(\mathbf{C}_{\mathbf{y} s}+\gamma \tilde{\mathbf{C}}_{\mathbf{y} s}\right)=\mathbf{C}_{\mathbf{y y}}^{-1}\left[\begin{array}{c}\mathbf{h} \\ \gamma \mathbf{h}^{*}\end{array}\right]$

For the proposed WL equalizer, the augmented autocorrelation matrix $\mathbf{C}_{\mathbf{y y}}$ expressed in (6) which gives a complete second order description of $\mathbf{r}^{\prime}$ is used for deriving the equalizer filter coefficient vector $\boldsymbol{\omega}_{n}$; whereas for the conventional linear MMSE algorithm, the filter coefficient vector $\mathbf{w}_{n}$ is calculated using only the autocorrelation of the observation $\mathbf{C}_{\mathbf{r}^{\prime} \mathbf{r}^{\prime}}=\mathrm{E}\left[\mathbf{r}_{n}^{\prime} \mathbf{r}_{n}^{\prime H}\right]$ and the cross-correlation between the observation and desired signal $\mathbf{C}_{\mathbf{r}^{\prime} s}=\mathrm{E}\left[\mathbf{r}_{n}^{\prime} s_{n}^{*}\right]$, i.e., $\mathbf{w}_{n}=\mathbf{C}_{\mathbf{r}^{\prime} \mathbf{r}^{\prime}}^{-1} \mathbf{C}_{\mathbf{r}^{\prime} s}$. The pseudoautocorrelation matrix $\tilde{\mathbf{C}}_{\mathbf{r}^{\prime} \mathbf{r}^{\prime}}$ is implicitly assumed to be zero. However, as analyzed earlier, $\tilde{\mathbf{C}}_{\mathbf{r}^{\prime} \mathbf{r}^{\prime}}$ is non-vanishing except at the initial stage for QPSK signals, hence omitting $\tilde{\mathbf{C}}_{\mathbf{r}^{\prime} \mathbf{r}^{\prime}}$ would lead to sub-optimum solutions. It is apparent from (6) that the complexity increase by applying WLP is due to the inverse operation of the $2 L \times 2 L$ matrix $\mathbf{C}_{\mathbf{y y}}$; whereas a conventional MMSE equalizer only involves an inverse operation of the $L \times L$ matrix $\mathbf{C}_{\mathbf{r}^{\prime} \mathbf{r}^{\prime}}$.

Next, we explain how LLR values for $x_{n}$ and $y_{n}$ are derived for QPSK systems based on the WL filter output so that the interference cancellation and iterative process can be carried out. The derivation for BPSK systems can be conducted similarly. It was shown in [9], [10] that the MMSE filter output $z_{n}$ can be well approximated as a Guassian random variable, i.e., $z_{n}=\mu s_{n}+\mu^{\prime} s_{n}^{*}+\eta$ where $\eta \sim \mathcal{N}\left(0, N_{\eta}\right)$. The real-valued parameters $\mu, \mu^{\prime}, N_{\eta}$ can be determined by taking expectation with respect to the interfering symbols and the channel noise

$$
\begin{aligned}
\mu= & \mathrm{E}\left\{z_{n} s_{n}^{*}\right\}=\boldsymbol{\omega}_{n}^{H} \mathrm{E}\left[\mathbf{y} s_{n}^{*}\right]=\boldsymbol{\omega}_{n}^{H} \mathbf{C}_{\mathbf{y} s} ; \\
\mu^{\prime}= & \mathrm{E}\left\{z_{n} s_{n}\right\}=\boldsymbol{\omega}_{n}^{H} \mathrm{E}\left[\mathbf{y} s_{n}\right]=\boldsymbol{\omega}_{n}^{H} \tilde{\mathbf{C}}_{\mathbf{y} s} ; \\
N_{\eta}= & \mathrm{E}\left[|\eta|^{2}\right]=\mathrm{E}\left[\left|z_{n}-\mu s_{n}-\mu^{\prime} s_{n}^{*}\right|^{2}\right] \\
= & \mathrm{E}\left\{\left|z_{n}\right|^{2}\right\}-\mu \mathrm{E}\left[z_{n} s_{n}^{*}\right]-\mu \mathrm{E}\left[z_{n}^{*} s_{n}\right]+\mu^{2} \\
& -\mu^{\prime} \mathrm{E}\left[z_{n} s_{n}\right]-\mu^{\prime} \mathrm{E}\left[z_{n}^{*} s_{n}^{*}\right]+\mu^{\prime 2} \\
= & \mathrm{E}\left\{\left|z_{n}\right|^{2}\right\}-\mu^{2}-\mu^{\prime 2}=\mu+\gamma \mu^{\prime}-\mu^{2}-\mu^{\prime 2} .
\end{aligned}
$$

The above equation holds since $z_{n}=\boldsymbol{\omega}_{n}^{H} \mathbf{y}$ and $\boldsymbol{\omega}_{n}=$ $\mathbf{C}_{\mathbf{y y}}^{-1}\left(\mathbf{C}_{\mathbf{y} s}+\gamma \tilde{\mathbf{C}}_{\mathbf{y} s}\right)$. Therefore,

$$
\begin{aligned}
\mathrm{E}\left\{\left|z_{n}\right|^{2}\right\} & =\mathrm{E}\left\{\boldsymbol{\omega}_{n}^{H} \mathbf{y} \mathbf{y}^{H} \boldsymbol{\omega}_{n}\right\}=\boldsymbol{\omega}_{n}^{H} \mathbf{C}_{\mathbf{y y}} \boldsymbol{\omega}_{n} \\
& =\left(\mathbf{C}_{\mathbf{y} s}^{H}+\gamma \tilde{\mathbf{C}}_{\mathbf{y} s}^{H}\right) \mathbf{C}_{\mathbf{y y}}^{-1} \mathbf{C}_{\mathbf{y y}} \boldsymbol{\omega}_{n}=\mu+\gamma \mu^{\prime} .
\end{aligned}
$$

For QPSK signal constellation with transmitted symbol $s_{n}=$ $\frac{ \pm}{\sqrt{2}}+\frac{ \pm j}{\sqrt{2}}$, it can be shown that the bit error rate (BER) can be expressed as

$$
P_{b}(\gamma)=\frac{1}{2}\left[Q\left(\frac{\mu(\gamma)+\mu^{\prime}(\gamma)}{\sqrt{N_{\eta}(\gamma)}}\right)+Q\left(\frac{\mu(\gamma)-\mu^{\prime}(\gamma)}{\sqrt{N_{\eta}}(\gamma)}\right)\right]
$$

where $Q(x)=\int_{x}^{\infty} \frac{1}{\sqrt{2 \pi}} \exp \left(-t^{2} / 2\right) d t$ is the complementary Gaussian cumulative distribution function. Note that in the above equation, $\mu, \mu^{\prime}$ and $N_{\eta}$ are all functions of $\gamma$ as can be seen from (8) and (9). The optimum values of $\gamma$ can be obtained by minimizing the $P_{b}(\gamma)$ expressed above. However, this minimum BER approach is not directly tractable. Alternatively, we can resort to numerical method to choose a proper value of $\gamma$, and the results presented in Section III indicate that the system performance is not sensitive to the choice of $\gamma$ if chosen in the right region.

After computing the values of $\mu, \mu^{\prime}$ and $N_{\eta}$, the conditional PDF of the equalizer output can be obtained as

$$
f\left(z_{n} \mid s_{m}\right)=\frac{1}{\pi N_{\eta}} \exp \left(-\frac{\left|z_{n}-\mu s_{m}-\mu^{\prime} s_{m}^{*}\right|^{2}}{N_{\eta}}\right) .
$$

and the LLR value of $x_{n}$ can thus be computed as

$$
\begin{aligned}
\lambda\left(x_{n}\right) & =\ln \frac{f\left(z_{n} \mid x_{n}=0\right)}{f\left(z_{n} \mid x_{n}=1\right)}=\ln \frac{f\left(z_{n} \mid s_{0}\right)+f\left(z_{n} \mid s_{3}\right)}{f\left(z_{n} \mid s_{1}\right)+f\left(z_{n} \mid s_{2}\right)} \\
& \approx \ln \frac{\exp \left(-\left|z_{n}-\mu s_{+}-\mu^{\prime} s_{+}^{*}\right|^{2} / N_{\eta}\right)}{\exp \left(-\left|z_{n}-\mu s_{-}-\mu^{\prime} s_{-}^{*}\right|^{2} / N_{\eta}\right)} \\
& =\frac{2}{N_{\eta}} \operatorname{Re}\left\{\left(\mu s_{+}^{*} z_{n}+\mu^{\prime} s_{+} z_{n}\right)-\left(\mu s_{-}^{*} z_{n}+\mu^{\prime} s_{-} z_{n}\right)\right\}
\end{aligned}
$$

where $s_{+}$denotes the QPSK symbol corresponding to $\max \left\{f\left(z_{n} \mid s_{0}\right), f\left(z_{n} \mid s_{3}\right)\right\}$, and $s_{-}$denotes the QPSK symbol corresponding to $\max \left\{f\left(z_{n} \mid s_{1}\right), f\left(z_{n} \mid s_{2}\right)\right\}$ since the real part of the symbols $s_{0}, s_{3}$ corresponds to 0 , and the real part of the symbols $s_{1}, s_{2}$ corresponds to 1 . The dual maxima rule [11] is used in (11) utilizing the fact that one term usually dominates each sum. Similarly,

$$
\begin{aligned}
\lambda\left(y_{n}\right) & =\ln \frac{f\left(z_{n} \mid s_{0}\right)+f\left(z_{n} \mid s_{1}\right)}{f\left(z_{n} \mid s_{2}\right)+f\left(z_{n} \mid s_{3}\right)} \\
& \approx \frac{2}{N_{\eta}} \operatorname{Re}\left\{\left(\mu s_{+}^{*} z_{n}+\mu^{\prime} s_{+} z_{n}\right)-\left(\mu s_{-}^{*} z_{n}+\mu^{\prime} s_{-} z_{n}\right)\right\}
\end{aligned}
$$

where $s_{+}$denotes the QPSK symbol corresponding to $\max \left\{f\left(z_{n} \mid s_{0}\right), f\left(z_{n} \mid s_{1}\right)\right\}$, and $s_{-}$denotes the QPSK symbol corresponding to $\max \left\{f\left(z_{n} \mid s_{2}\right), f\left(z_{n} \mid s_{3}\right)\right\}$ since the imaginary part of the symbols $s_{0}, s_{1}$ corresponds to 0 , and the imaginary part of the symbols $s_{2}, s_{3}$ corresponds to 1 .

\section{NUMERICAL RESULTS}

In this section, we compare the performance of the proposed scheme with that of the WL MMSE and DFE equalizers introduced in [4] and the iterative linear MMSE equalizer introduced in [8]-[10]. During each Monte-Carlo run, the block size is set to 10000 information bits, which corresponds to 5000 QPSK or 10000 BPSK symbols. The noise variance $N_{0}$ and path delays 


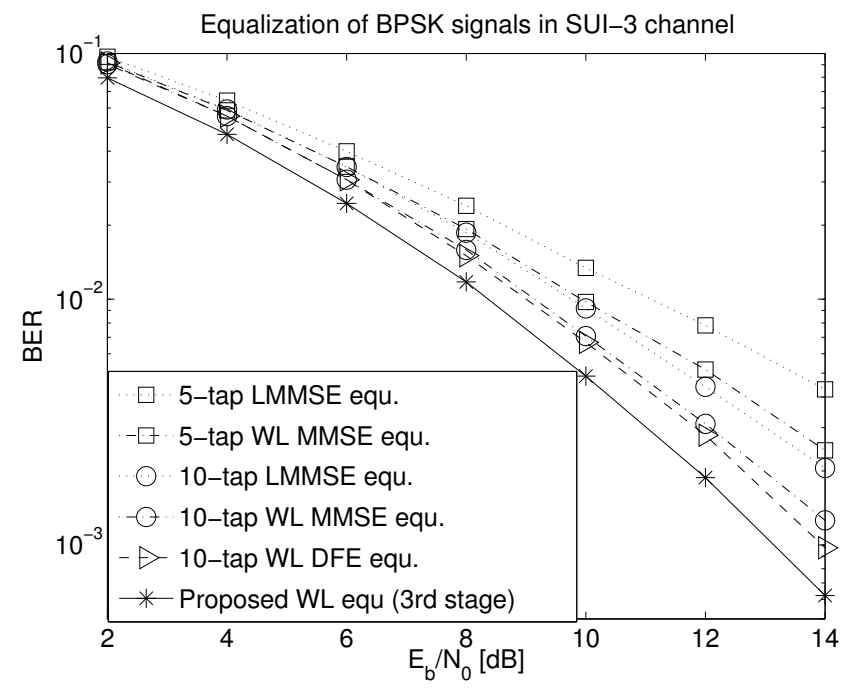

Fig. 1. Comparison of different equalization schemes for BPSK signals in SUI-3 channel.

as well as the complex channel coefficients are assumed to be known to the receiver. Both time-varying and static channels are tested. For the time-varying channel, we choose the SUI-3 fixed wireless access (FWA) channel introduced in [12]. The channel coefficients vary from one data block to another, however, they are assumed to remain constant during the transmission of one block of data. For the static channel, we use a 5-tap channel with impulse response $h[n]=(2-0.4 j) \delta[n]+(1.5+1.8 j) \delta[n-$ $1]+\delta[n-2]+(1.2-1.3 j) \delta[n-3]+(0.8+1.6 j) \delta[n-4]$. The total channel gain is normalized so that $P=\sum_{n=0}^{4}|h[n]|^{2}=1$.

Fig. 1 shows the performance of different equalization schemes for BPSK signals transmitted over the SUI-3 channel. The results are averaged over at least 500 channel realizations. The 5-tap and 10-tap WL MMSE equalizers proposed in [4] outperform their linear counterparts by over $1 \mathrm{~dB}$ at BERs between $10^{-2}$ and $10^{-3}$. The use of decision feedback can further improve the performance since it is observed that the 10tap WL DFE (6 feed-forward taps and 4 feedback taps) yields better results than the 10-tap WL MMSE. Equalizers with length greater than 10 taps are also tested and are shown to have a similar performance to the 10-tap equalizers. The figure also shows that the proposed equalizer at the 3rd stage outperforms the WL DFE by $0.7 \mathrm{~dB}$ at $\mathrm{BER}=10^{-3}$ (It is observed that most of the gains are obtained at the 2 nd and the 3rd stages with the proposed iterative equalization scheme).

Fig. 2 shows the impact of the parameter $\gamma$ on the performance of the proposed equalizer for QPSK signals transmitted over the SUI-3 channel. The curve is plotted for different values of $\gamma$ at the 5th equalization stage, and $E_{b} / N_{0}$ is set to $20 \mathrm{~dB}$. The figure shows that the choice of $\gamma=1$ leads to the worst performance. This is in contrast to systems with real-valued constellations, for which $\gamma=1$ is appropriate. The reason is that $\boldsymbol{\omega}_{n}^{2}=\boldsymbol{\omega}_{n}^{1 *}$ [3], [4] when choosing $\gamma=1$, and it can be shown that $\mu=\mu^{\prime}$ in such a case. Therefore, the filter output becomes $z_{n}=\mu s_{n}+\mu^{\prime} s_{n}^{*}+\eta=2 \mu \operatorname{Re}\left\{s_{n}\right\}+\eta$, which means the decision statistic does not contain any information about the imaginary part of the symbol $s_{n}$. Consequently, the second bit corresponding to the imaginary part of the QPSK symbol cannot be correctly detected. As indicated by Fig. 2, there is

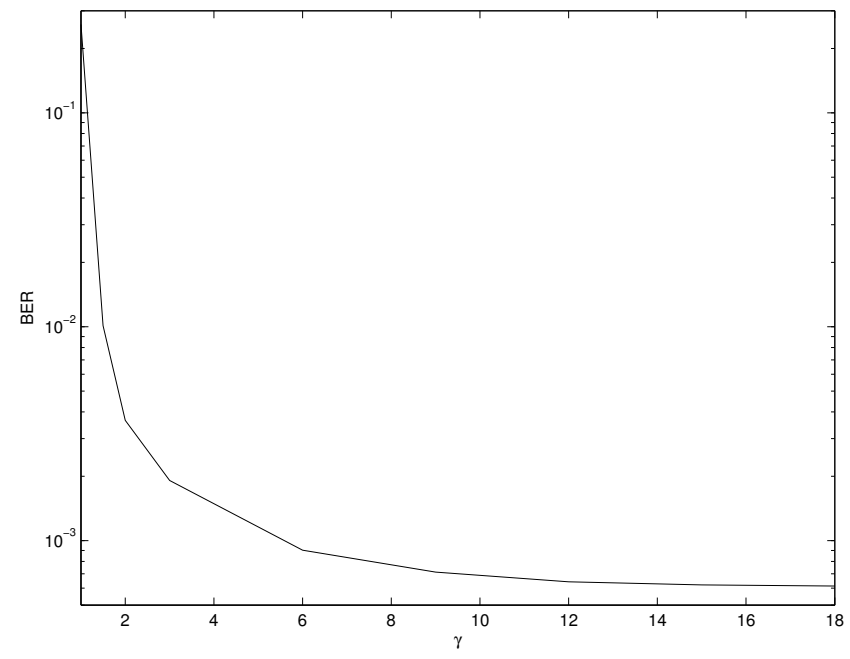

Fig. 2. BER as a function of $\gamma$.

a dramatic improvement in BER performance when increasing the value of $\gamma$ from 1 to 6 , beyond which the performance becomes insensitive to the choice of $\gamma$. The value of $\gamma$ is set to 15 for the rest of the experiments.

Fig. 3 shows the performance comparison between the proposed scheme and the iterative linear MMSE scheme proposed in [8]-[10] for QPSK signals transmitted over the SUI-3 channel. The topmost curve represents initial stage equalization and the bottommost curve represents the 6th stage equalization. It takes 5 stages for both algorithms to converge. Upon convergence, the proposed scheme outperforms the linear MMSE scheme by over $4 \mathrm{~dB}$ at $\mathrm{BER}=10^{-3}$. Compared to the initial equalization stage, the subsequent equalization stages achieve much better performance. However, most gains are obtained at the 2nd and 3rd stages. As expected, the two algorithms have identical performance at the initial stage, since $\widetilde{\mathbf{C}}_{\mathbf{r}^{\prime} \mathbf{r}^{\prime}}=\mathbf{0}$ for QPSK signalling at the initial stage, thus no improvement can be achieved by WLP. At the following cancellation stages, the ISI canceled signal becomes improper as shown in Section II, and exploitation of the improperness of the ISI results in better performance. The two schemes are compared for the 5-tap static channel in Fig. 4. After the system reaches convergence at the 6th stage, a performance gain of $1 \mathrm{~dB}$ is observed by applying WLP at BER $=10^{-4}$. As indicated by Fig. 3 and 4 , the proposed equalizer does not achieve much gain at low SNR. This is due to the fact that at low SNR, the proper noise is dominant; whereas the advantage of exploiting the improper ISI becomes evident when SNR increases.

The proposed scheme can be easily extended to a coded system to design an iterative equalization and decoding scheme (turbo equalization) as in [8]-[10]. However, our observation is that the use of WLP in a coded system does not lead to as much gain as in an uncoded system for the investigated ISI channels. The reason is that BER performance of a coded system is much better than that of an uncoded system. Consequently, the reliability value $\lambda\left(s_{n}\right)$ in a coded system can become very high, leading to $\bar{s}_{n}=\tanh \left[\lambda\left(x_{n}\right) / 2\right] / \sqrt{2}+j \tanh \left[\lambda\left(y_{n}\right) / 2\right] / \sqrt{2} \approx$ $\pm 1 / \sqrt{2} \pm j / \sqrt{2}$. Therefore, $\operatorname{Im}^{2}\left\{\bar{s}_{n}\right\}-\operatorname{Re}^{2}\left\{\bar{s}_{n}\right\} \approx 0$, and the pseudo-autocorrelation matrix approches zero $\left(\tilde{\mathbf{C}}_{\mathbf{r}^{\prime} \mathbf{r}^{\prime}}=\right.$ $\mathbf{H} \tilde{\mathbf{V}}_{n} \mathbf{H}^{T} \approx \mathbf{0}$ ), the gain obtained by WLP thus becomes small. 


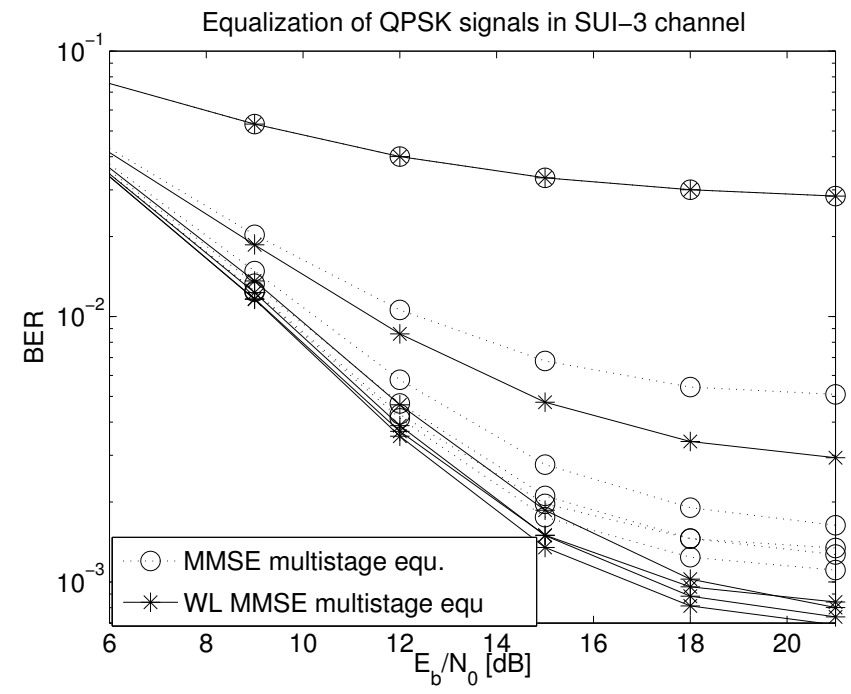

Fig. 3. Performance of the proposed equalization for QPSK signals in SUI-3 channel.

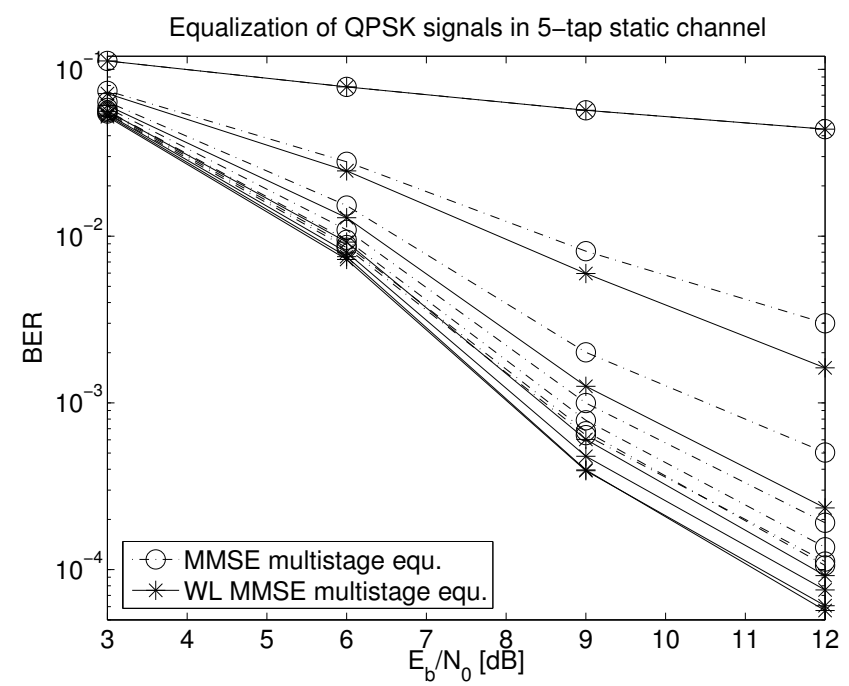

Fig. 4. Performance of the proposed equalization for QPSK signals in 5-tap static channel.

\section{Conclusions}

In this paper, we introduced a multistage widely linear equalization scheme for signals transmitted over frequency selective channels. The validity of employing WLP is justified by observing that the residual interference after cancellation is improper. By utilizing the improperness of the ISI canceled signal and by minimizing a revised cost function, we have come up with a new equalization scheme, which processes both the original and the complex-conjugated versions of the ISI canceled signals. The proposed algorithm is generic in the sense that it is applicable for both real and complex signalling formats. Simulations with BPSK and QPSK modulated systems without channel coding are shown and the results indicate that this approach leads to a performance that is superior to the conventional linear MMSE equalizers at the cost of increased complexity. Unfortunately, applying a similar procedure to a coded system employing iterative equalization and decoding does not yield significant performance gain.

\section{REFERENCES}

[1] P. Schreier, L. Scharf, C. Mullis. "Detection and estimation of improper complex random signals", IEEE Transactions on Information Theory, vol. 51, no. 1, pp. 306-312, January 2005.

[2] F. Neeser, J. Massey. "Proper complex random processes with applications to information theory", IEEE Transactions on Information Theory, vol. 39, no. 4, pp. 1293-1302, July 1993.

[3] B. Picinbono, P. Chevalier. "Widely linear estimation with complex data", IEEE Transactions on Signal Processing, vol. 43, no. 8, pp. 2030-2033, August 1995.

[4] W. Gerstacker, R. Schober, A. Lampe. "Receivers with widely linear processing for frequency-selective channels". IEEE Transactions on Communications, vol. 51, no. 9, pp. 1512-1523, Sept. 2003.

[5] W. Gerstacker, F. Obernosterer, R. Schober, A. Lehmann, A. Lampe, P. Gunreben. "Equalization concepts for Alamouti's space-time block code", IEEE Transactions on Communications, vol. 52, no. 7, pp. 1178-1190, July 2004.

[6] D. Mattera, L. Paura, F. Sterle. "Widely linear MMSE equaliser for MIMO linear time-dispersive channel", Electronic Letters, vol. 39, no. 20, pp. 1481-1482, Oct. 2003.

[7] D. Mattera, L. Paura, F. Sterle. "Widely linear decision-feedback equalizer for time-dispersive linear MIMO channel", IEEE Transactions on Signal Processing, vol. 53, no. 7, pp. 2525-2536, July 2005.

[8] K. Narayanan. "Turbo equalization". In Wiley Encyclopedia of Telecommunications, vol. 5, pp. 2716-2727, 2002.

[9] X. Wang, H. Poor. "Iterative (turbo) soft interference cancellation and decoding for coded CDMA", IEEE Transactions on Communications, vol. 47, pp. 1046-1061, July 1999

[10] M. Tuchler, R. Koetter, A. Singer. "Turbo equalization: principles and new results", IEEE Transactions on Communications, vol. 50, pp. 754767, May 2002.

[11] A. Viterbi. "An intuitive justification and a simplified implementation of the MAP decoder for convolutional codes". IEEE Journals on Selected Areas in Communications, vol. 16, no. 2, pp. 260-264, Feb. 1998.

[12] Channel Models for Fixed Wireless Applications. available at http://www.esqube.com/ieee802.16channelModels.pdf 